\title{
EFFECTS OF OPERATIONAL VARIABLES ON THE PULP YIELD AND LIGNIN DISSOLUTION OF CYPERUS ARTICULATUS
}

\author{
B.O. Ogunsile \\ Department of Chemistry, University of Ibadan, Ibadan
}

\begin{abstract}
S
The influence of operational variables on the pulp yield and lignin dissolution of Cyperus articulatus was studied. The study was aimed at optimizing pulping variables and selecting suitable delignification conditions for the production of pulp and paper from the plant. Cyperus articulatus chips were delignified in a laboratory pulpwood digester under the soda pulping process using the following pulping conditions: time (30 - 150 minutes), temperature $\left(120-150^{\circ} \mathrm{C}\right)$, liquor to solid ratio (10:1 - 20:1) and concentration of cooking liquor (4 - 6\% caustic soda). Experimental data were fitted into a central composite design and the polynomial equation derived reproduced the pulp yields and the residual lignin contents with errors less than 6\%. Optimum pulp yields were obtained using low values of the process variables while the reverse was the case for the residual lignin. Pulping at high temperatures for a short cooking time with low to medium concentration of pulping liquor gives the best compromise for both pulp yield and residual lignin content.
\end{abstract}

Keywords: Cyperus articulatus, soda pulping, pulp yield, residual lignin.

\section{INTRODUCTION}

Paper can be produced from virtually any cellulose containing material. Cellulose, one of the most abundant organic substances available, forms the major component of most plants and is constantly replaceable. In the manufacture of pulp and paper, cellulose fibres are liberated from the matrix of lignin which cements them together. Fiber liberation can be achieved by heat, mechanical, chemical, biochemical means or a combination of any of these (Haygreen and Bowyer, 1982).

The mechanical process involves both com- pression and friction of wood chips or logs which produces heat that softens the lignin thus releasing the fibers. In some processes, the wood chips are preheated before the mechanical treatment (Casey, 1960; James and Raymond, 1970). The paper produced in the mechanical process is weak and non-permanent because it contains high lignin and relatively little cellulose.

In the chemical pulping, chemical reagents are used to effect separation of the cellulose fibers from the lignin matrix and other components (Gierer, 1985). In principle, wood chips are 


\section{Ogunsile}

digested with suitable chemicals at elevated temperature and pressure using appropriate liquor to solid ratio. The chemical process has comparative advantage over the mechanical process in that the paper produced is stronger and permanent because of its low lignin content. The successful operation of the chemical pulping processes depend on certain operational variables such as time, temperature, concentration of cooking liquor, chip size and liquor to solid ratio (Iglesias et al., 1996; Jime'nez et al., 2008). The selection of suitable delignification conditions and optimization of process variables are therefore highly essential for pulp and paper production by the chemical pulping processes. Kinetic modeling based on mathematical design have been used as a way of optimizing operating condition in pulping processes (Tjeerdsma et al., 1994). However, this model becomes too complex when more than two independent variables are involved. A more recent approach is the use of central composite factorial design (Aknazarova and Kafarov, 1982). The factor design is preferred to kinetic modeling because of its simplicity especially when several variables are involved. It allows the development of empirical models involving several independent variables in order to identify patterns of variation in the dependent variables of various pulping processes as applied to diverse vegetable materials (Vazquez et al., 1995; Vega et al., 1997; Gilarianz et al., 1999; Jimenez et al., 2000, Jimenez et al., 2008)

Cyperus articulatus is a non-wood mat-making plant whose fiber characteristics and soda pulping was reported recently (Ogunsile et al., 2007). The present study was aimed at evaluating the influence of operating variables on the pulp yield and lignin dissolution of this plant using a central composite factorial design.

MATERIALS AND METHODS

Cyperus articulatus was collected along the riverine area of Ikorodu in Lagos state. The plant was manually cut into chips of about 2$4 \mathrm{~cm}$ long, sun-dried and stored at room temperature in a polythene bag. Soda cooking liquor was prepared from a standard concentrated solution of sodium hydroxide by serial dilution with de-ionized water. The plant samples were pulped in a 10-litre electrically heated, thermostatically controlled stainless steel digester. Chips from the Cyperus articulatus were weighed and charged into the digester with the required amount of chemical solution at liquor to solid ratio of 10:1 and 20:1. The digester was heated to the operating temperatures $\left(120^{\circ} \mathrm{C}\right.$ and $\left.150^{\circ} \mathrm{C}\right)$ and time (30 - 150 minutes), which was then maintained throughout the experiment. The resulting pulp was thoroughly washed with tap water and the pulp yield was determined gravimetrically after drying at $102^{\circ} \mathrm{C}$ to constant weight in the oven. The pulps were analyzed for Kappa number as described in TAPPI standard (1993). The residual lignin was estimated from the Kappa number by multiplying with a factor of 0.13 (TAPPI, 1993).

\section{The central composite factorial design}

The central composite factorial design was employed to evaluate and quantify the effect of the operational variables on the pulp yields and residual lignin. The effect of the variables were quantified more precisely by choosing part of the experimental results and grouping them to form a first order full factorial design, with variables at two levels $\left(2^{4}\right)$. Using this design, some of the experimental data were fitted to a first order polynomial regression equation as implemented in the "SPSS" statistical package. Individual and second order interaction influences over the response surface of the independent variables were evaluated (Aknazarova and Kafarov, 1982).

The mathematical model was:

$$
\begin{aligned}
Y= & a_{0}+a_{1} X_{1}+a_{2} X_{2}+a_{3} X_{3}+a_{4} X_{4}+a_{12} X_{1} X_{2}+a_{13} X_{1} X_{3}+a_{14} X_{1} X_{4}+a_{23} X_{2} X_{3} \\
& +a_{24} X_{2} X_{4}+a_{34} X_{3} X_{4}
\end{aligned}
$$


The response variable $\mathrm{Y}$ represents the pulp yield and residual lignin. The independent variables $X_{1}, \mathrm{X}_{2}, \mathrm{X}_{3}$, and $\mathrm{X}_{4}$ correspond to liquor to solid ratio LS, time, temperature and concentration of cooking liquor respectively. The ranges of values for each independent variable were: LS, $10: 1$ to $20: 1$, time, 30-150 minutes, temperature, $120-150^{\circ} \mathrm{C}$ and concentration, $4-6 \%$ $\mathrm{NaOH}$ solution.

The values of the independent variables were normalized from -1 to +1 by using the equation

$X_{n}=\frac{2(\bar{X}-X)}{\left(X_{\max }-X_{\min }\right)}$

Where:

$X_{n}$ is the normalized value of LS, time, temperature and concentration,

$X$ is the absolute experimental value of the vari-

$\bar{X}$ is the mean of all the experimental values for the variable in question

$X_{\max }$ and $X_{\min }$ are the maximum and minimum values respectively of such a variable.

\section{RESULTS AND DISCUSSION}

The composition of the sun-dried sample was determined to be $40.9 \%$ cellulose, $24.3 \%$ lignin and $6.9 \%$ ash. The alcohol-benzene extractives, cold water solubles, hot water solubles, and $1 \%$ $\mathrm{NaOH}$ solubles were $6.52 \%, 12.03 \%$, and $26.6 \%$ respectively. The fiber was $1.5 \mathrm{~mm}$ long, $22.2 \mu \mathrm{m}$ wide and $6.1 \mu \mathrm{m}$ thick (Ogunsile et al., 2007). The experimental design $\left(2^{4}\right.$ factorial designs) together with the pulp yield and the residual lignin are presented in Table 1. Experiments 1-16 allowed the calculation of different parameters in the regression equations $a_{i}$ and $a_{i j}$. These were subsequently subjected to a $\mathrm{T}$ - test to check their significance at 90 to $95 \%$ confidence level using the experimental error estimated from the replication at the central point of the design, that is, experiments 17,18 and 19 of Table 1 . The values of experiment 12 was corrected by extrapolation. The central point of the design corresponds to the following reaction conditions:

Liquor to solid ratio, $\mathrm{LS}=15: 1$,

Time $=90$ minutes,

Temperature $=135^{\circ} \mathrm{C}$

Concentration $=5 \%$ soda

Table 1: Experimental design and result for yield and residual lignin

\begin{tabular}{|c|c|c|c|c|c|c|}
\hline Experiment & $\begin{array}{l}\text { LS } \\
X_{1}\end{array}$ & $\begin{array}{c}\text { Time } \\
\mathrm{X}_{2}\end{array}$ & $\begin{array}{c}\text { Temperature } \\
X_{3}\end{array}$ & $\begin{array}{c}\text { Concentration } \\
X_{4}\end{array}$ & $\begin{array}{l}\text { Yield } \\
(\%)\end{array}$ & $\begin{array}{c}\text { Lignin } \\
(\%)\end{array}$ \\
\hline 1 & -1 & -1 & -1 & -1 & 30.2 & 4.31 \\
\hline 2 & 1 & -1 & -1 & -1 & 27.7 & 4.00 \\
\hline 3 & -1 & 1 & -1 & -1 & 27.8 & 3.65 \\
\hline 4 & 1 & 1 & -1 & -1 & 25.0 & 3.28 \\
\hline 5 & -1 & -1 & 1 & -1 & 26.6 & 3.39 \\
\hline 6 & 1 & -1 & 1 & -1 & 24.4 & 3.50 \\
\hline 7 & -1 & 1 & 1 & -1 & 21.8 & 1.73 \\
\hline 8 & 1 & 1 & 1 & -1 & 21.4 & 1.92 \\
\hline 9 & -1 & -1 & -1 & 1 & 28.6 & 3.26 \\
\hline 10 & 1 & -1 & -1 & 1 & 26.9 & 2.96 \\
\hline 11 & -1 & 1 & -1 & 1 & 23.8 & 2.70 \\
\hline 12 & 1 & 1 & -1 & 1 & 20.5 & 1.63 \\
\hline 13 & -1 & -1 & 1 & 1 & 27.3 & 2.69 \\
\hline 14 & 1 & -1 & 1 & 1 & 24.4 & 2.58 \\
\hline 15 & -1 & 1 & 1 & 1 & 21.0 & 2.60 \\
\hline 16 & 1 & 1 & 1 & 1 & 20.7 & 1.69 \\
\hline 17 & 0 & 0 & 0 & 0 & 27.7 & 2.01 \\
\hline 18 & 0 & 0 & 0 & 0 & 28.3 & 1.96 \\
\hline 19 & 0 & 0 & 0 & 0 & 27.8 & 1.93 \\
\hline
\end{tabular}




\section{Ogunsile}

All normalized independent variables for the central points of the design are zero.

Table 2: Signicative regression parameters

\begin{tabular}{lrc}
\hline & Yield & Residual Lignin \\
\hline$\alpha_{0}$ & 27.93 & 1.97 \\
$\alpha_{1}$ & -1.01 & -0.17 \\
$\alpha_{2}$ & -2.13 & -0.47 \\
$\alpha_{3}$ & -1.43 & -0.36 \\
$\alpha_{4}$ & -0.73 & 0.35 \\
$\alpha_{12}$ & $(-0.16)$ & $(-0.10)$ \\
$\alpha_{13}$ & $(-0.28)$ & $(-0.08)$ \\
$\alpha_{14}$ & $(-0.02)$ & $(-0.13)$ \\
$\alpha_{23}$ & $(-0.09)$ & $(-0.06)$ \\
$\alpha_{24}$ & -0.52 & $(-0.11)$ \\
$\alpha_{34}$ & 0.63 & 0.23 \\
$\alpha_{44}$ & -3.05 & 0.9 \\
\hline
\end{tabular}

The non-significant parameters at 0.1 levels are in parenthesis
The coefficients of the model equations and the statistical parameters establishing their validity are summarized in Table 2 . The dependent variables (i.e. pulp yield and residual lignin) were related to the independent variables through the following equations:

Yield $=27.93-1.01 X_{1}-2.13 X_{2}-1.43 X_{3}-0.73 X_{4}$

$-0.52 X_{2} X_{4}+0.63 X_{3} X_{4}-3.05 X_{4}^{2}$

Residual Lignin $\mathrm{n}=1.97-0.17 X_{1}-0.47 X_{2}$

$-0.36 X_{3}-0.35 X_{4}+0.23 X_{3} X_{4}+0.9 X_{4}^{2}$

Values calculated from the respective polynomial equations above were plotted with the experimental results for the different response variables as shown in Figures $1 \mathrm{a}$ and $\mathrm{b}$.

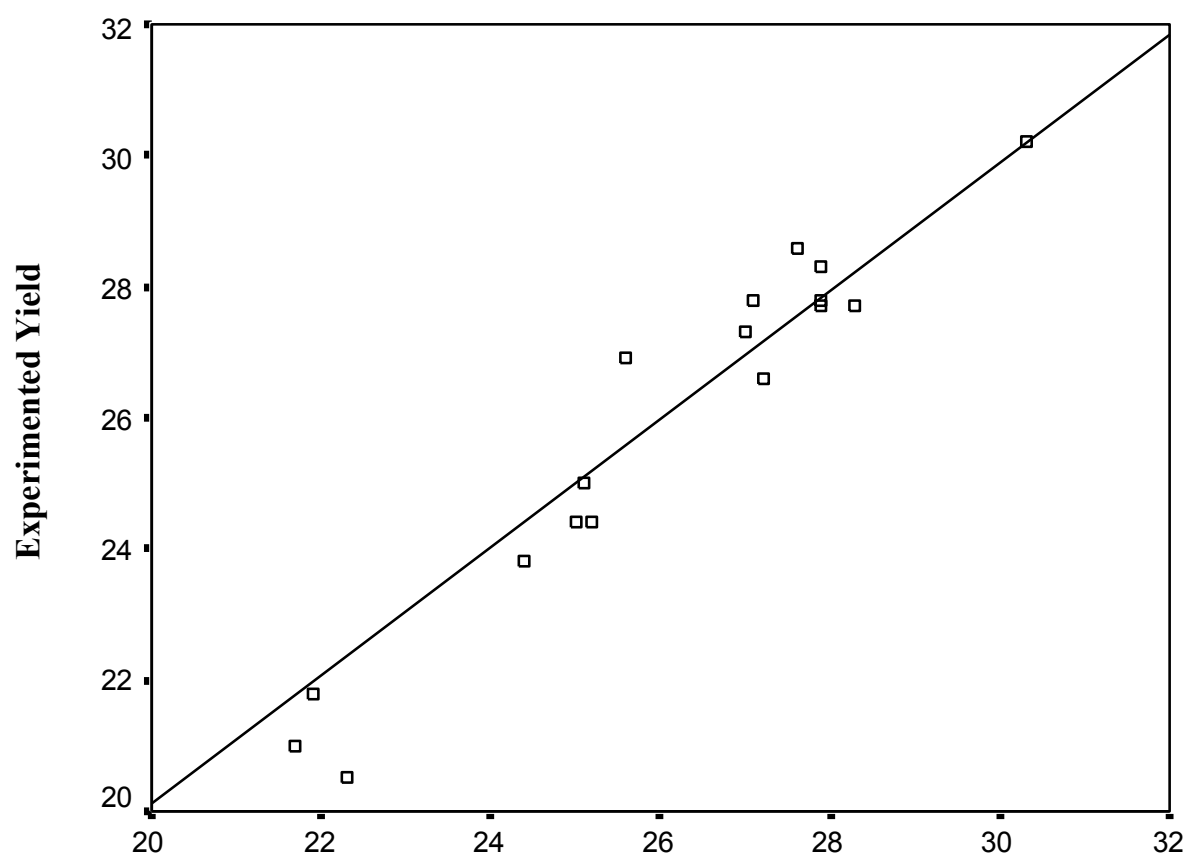

Calculated Yield

Fig. 1a: Correlation between experimental and calculated values of (a) pulp yield 


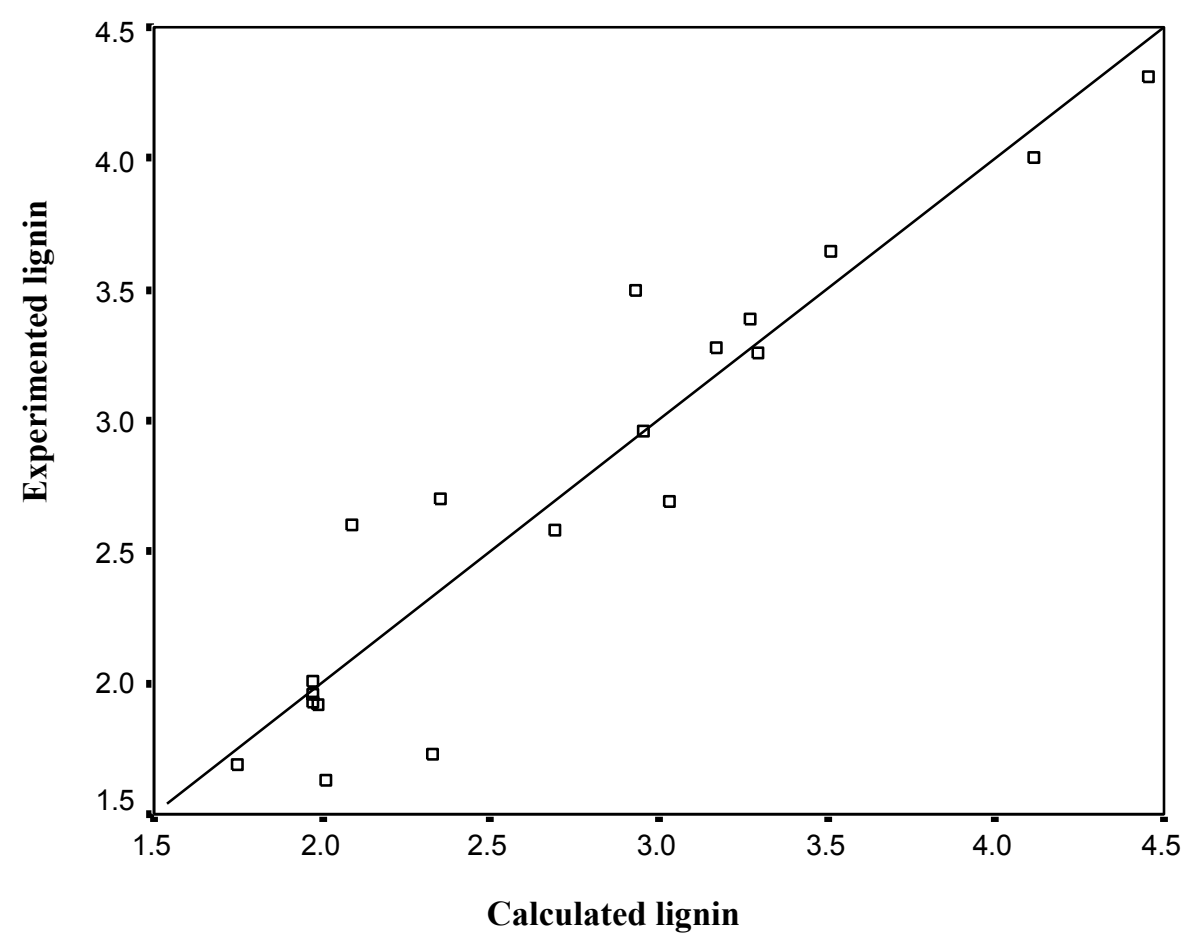

Fig. 1b: Correlation between experimental and calculated values of ((b) residual lignin

The results showed good correlation between the experimental values and those predicted by the models.

Pulp yield: Equation 3 allows the estimation of the variation of the yield with changes in each independent variable over the range considered while the other three remain constant. Non-significant parameters were dropped to obtain simpler equations. The equation showed that all the operation variables, namely, the LS, time, concentration and temperature had significant influence at $90 \%$ confidence level on the pulp yield. Also, on the pulp yield, there were significant interactions between time and concentration on one hand and temperature and concentration of the cooking liquor on the other hand. The lowest pulp yield $(19.7 \%)$ was obtained at large values of the four process variables (i.e. +1 for all). If yield similar to chemical pulp is desired, then all the process vari- ables must be at normalized values of +1 . However, this lowest yield can be raised to $25 \%$ by using a lower pulping time ( -1 normalized value). Pulping at a lower time will reduce the degradation effects of temperature and pulping liquor on the cellulose. This will also result in some energy savings in addition to increasing the pulp yield. The highest obtainable yield was $30.3 \%$, which occurs at low values of the process variables ( -1 for all). The maximum and minimum variations in the highest pulp yield were caused by changes in time (a change of 5.3 units) and concentration (a change of 0.2 unit) respectively, the effects of temperature (2.64 units) and LS (2.01 units) lie in between.

Delignification. The minimum lignin content, according to equation 4 , was obtained at high values of cooking time, temperature and cooking liquor $(+1$ for all). Under this condition, the 


\section{Ogunsile}

maximum variation in the minimum residual lignin value was caused by a change in pulping time (0.94 units) followed by LS, temperature and concentration respectively. The lowest residual lignin content $(1.73 \%)$ can be decreased further to $1.36 \%$ by setting the concentration to a value of +0.5 unit. Pulping at high temperatures for a short cooking time with low to relatively high concentration of pulping liquor would give the best compromise for both pulp yield and residual lignin content.

Ratio of dissolved lignin to weight losses. The ratio of the dissolved lignin to the weight losses as presented in Table 3 can be used to estimate the quantity of lignin present in the pulp provided the pulp yield is known. The result showed that the values were relatively constant irrespective of the duration or time of cooking. The average ratio of the dissolved lignin to the weight loss was 0.13 (Table 3 ) compared to a value of about 0.4 reported for Miscanthus sinensis (Iglesias et al., 1996). The differences in the values may be attributed to the nature of the material concerned, the amount of lignin present and the ease of lignin dissolution. The constant values seem to suggest that solubilization and delignification take place at the same rate.

\section{CONCLUSION}

The effects of operational variables on the pulp yield and residual lignin were evaluated using a central composite model. The equation showed that the LS, time and temperature had significant influence on the pulp yield while concentration of the cooking liquor did not. The lowest pulp yield was obtained at large values of the four process variables. Pulping at a lower time will reduce the degradation effects of temperature on the cellulose. This will also result in some energy savings in addition to increasing the pulp yield. The maximum and minimum variations in the highest pulp yield were caused by changes in time and concentration, respectively. The minimum lignin content was obtained at high values of the operational variables. Pulping at high temperatures for a short cooking time with low to relatively high concentration of pulping liquor gives the best compromise for both pulp yield and residual lignin content. The ratios of the dissolved lignin to the weight losses were relatively constant irrespective of the duration or time of cooking.

\section{ACKNOWLEDGEMENT}

The author wishes to acknowledge the technical assistance rendered by Messrs Rasaq, I.A. and O.M. Anifowose of the Department of Chemistry, University of Ibadan.

\section{REFERENCES}

Aknazarova, S. and Kafarov, V. (1982). Experiment Optimization in Chemistry and Chemical Engineering. Mir Publishers, Moscow.

Casey, P.J. (1960). Pulp and paper: Chemistry and chemical technology, $2^{\text {nd }}$ Edition, Vol. 1: Pulping and bleaching. Interscience Publisher and Inc. New York, 694pp.

Gierer, J. (1985). Chemistry of delignification, Part 1: General concept and reactions during pulping. Wood Science and Technology. 19, 289-312.

Table 3: Ratio of dissolved lignin to weight losses

\begin{tabular}{lcccccc}
\hline Cooking liquors & $\begin{array}{c}\text { Temperature } \\
\left({ }^{\mathbf{0}} \mathbf{C}\right)\end{array}$ & $\mathbf{3 0}$ & $\mathbf{6 0}$ & \multicolumn{2}{c}{ Time (minutes) } & \multirow{2}{*}{$\mathbf{9 0}$} \\
\hline \multirow{2}{*}{ 4\% Soda } & 120 & 0.12 & 0.12 & 0.12 & 0.13 & 0.12 \\
& 150 & 0.12 & 0.13 & 0.13 & 0.13 & 0.14 \\
& & & & & & \\
\multirow{2}{*}{ 6\% Soda } & 120 & 0.13 & 0.13 & 0.13 & 0.13 & 0.13 \\
& 150 & 0.13 & 0.13 & 0.13 & 0.13 & 0.12 \\
\hline
\end{tabular}

Journal of Science and Technology @ KNUST April 2010 
Gilarranz , M.A., Oliet, M., Rodriguez , F. and Tijero J. (1999) . Methanol based pulping of Eucalytus globulus, Canadian J. Chemical Engineering. 77 (3), 515-521.

Heygreen, J.G. and Bowyer, J.L. (1982). Forest Products and Wood Science: An Introduction. The Iowa State University Press/ AMES, pp. 3379-3418.

Iglesias, G., Bao, M., Lamas J., and Vega, A. (1996). Soda pulping of Miscanthus sinensis: Effects of operational variable on pulp yield and lignin solubilization. Biores. Technol., 58, 17-23.

James, H.P. and Raymond, A.L. (1970). Ground wood - Stone groung wood chips. In Britt K.W. Handbook of pulp and paper technology, van Nostrand Reinhold Company, New York, pp. 179-196.

Jimenez, L. Pe'rez, A., DelaTorre, M.J., Rodri'guez, A. and Angulo, V. (2008). Ethyleneglycol pulp from tagasaste. Bioresource Technology, 99, 2170-2176.

Jimenez, L., Perez, I., DelaTorre, M.J., Lopez F. and Arisa, J. (2000). Use of formaldehyde for making wheat straw cellulose pulp. Bioresource Technology, 72, 283-288.
Ogunsile, B.O., Rasaq, I.A. and Anifowose, O.M. (2007). Fiber morphology and soda pulp from Cyperus articulatus. International Journal of Chemistry, Vol. 17(1), 16.

Technical Association of pulp and paper Industry (TAPPI). (1993). Kappa number of pulp T236CM -85 .

Tjeerdsma, B.F., Zomers, F.H.A., Wilkizon, E.C. and Sierra-Alvarez, R. (1994). Modeling organosolv pulping of hemp. Holzforschung. 48 (5), 415 - 422.

Vazquez, G., Antorrena, G. and Gonzalex, J. (1995). Acetosolv pulping of Eucalyptus globulus wood. 1. The effect of operational variables on pulp yield, pulp lignin content and pulp potential glucose content. Holzforschung. 49 (1), 69-74.

Vega , A., Bao, M. and Lamas, J. (1997). Application of factorial design to the modelling of organosolv delignification of Miscanthus sinensis (elephant grass) with phenol and dilute - acid solutions. Bioresource Technology 61(1), 1-7. 